\title{
Development of Fatty Acid Reference Ranges and Relationship with Lipid Biomarkers in Middle-Aged Healthy Singaporean Men and Women
}

\author{
Cody A. C. Lust ${ }^{1}$, Xinyan Bi ${ }^{2}$, Christiani Jeyakumar Henry ${ }^{2,3}$ and David W. L. Ma ${ }^{1, *(D)}$ \\ 1 Department of Human Health and Nutritional Sciences, University of Guelph, Guelph, ON N1G 2W1, Canada; \\ clust@uoguelph.ca \\ 2 Clinical Nutrition Research Centre (CNRC), Singapore Institute of Food and Biotechnology Innovation (SIFBI) \\ Agency for Science, Technology and Research (A*STAR), 14 Medical Drive \#07-02, MD 6 Building, \\ Singapore 117599, Singapore; bi_xinyan@sifbi.a-star.edu.sg (X.B.); jeya_henry@sifbi.a-star.edu.sg (C.J.H.) \\ 3 Department of Biochemistry, Yong Loo Lin School of Medicine, National University of Singapore, \\ Singapore 117599, Singapore \\ * Correspondence: davidma@uoguelph.ca
}

Citation: Lust, C.A.C.; Bi, X.; Henry, C.J.; Ma, D.W.L. Development of Fatty Acid Reference Ranges and Relationship with Lipid Biomarkers in Middle-Aged Healthy Singaporean Men and Women. Nutrients 2021, 13, 435. https://doi.org/10.3390/ nu13020435

Received: 8 December 2020

Accepted: 26 January 2021

Published: 29 January 2021

Publisher's Note: MDPI stays neutral with regard to jurisdictional claims in published maps and institutional affiliations.

Copyright: (c) 2021 by the authors. Licensee MDPI, Basel, Switzerland. This article is an open access article distributed under the terms and conditions of the Creative Commons Attribution (CC BY) license (https:// creativecommons.org/licenses/by/ $4.0 /)$.

\begin{abstract}
Dietary fatty acids (FA) are essential for overall human health, yet individual FA reference ranges have yet to be established. Developing individual FA reference ranges can provide context to reported concentrations and whether an individual displays deficient, or excess amounts of FA. Reference ranges of sixty-seven individual FA $(\mu \mathrm{mol} / \mathrm{L})$ were profiled and analyzed using gas chromatography with a flame ionization detector from serum samples collected from 476 middle-aged Singaporean males (BMI:23.3 \pm 2.9 ) and females (BMI:21.8 \pm 3.6 ). Measures of triglycerides (TG), high-density lipoprotein (HDL), low-density lipoprotein (LDL), and total cholesterol (TC) (mmol/L) were also collected. The mean FA concentration seen in this cohort $(11,458 \pm 2478$ was similar to that of overweight North American cohorts assessed in past studies. Ten biologically relevant FA were compared between sexes, with females exhibiting significantly higher concentrations in four FA $(p<0.05)$. A multiple regression model revealed the ten FA contributed significantly to nearly all lipid biomarkers $(p<0.05)$. A majority of participants who had FA concentrations in the $\geq 95$ th percentile also exhibited TG, HDL, LDL, and TC levels in the "high" risk classification of developing cardiovascular disease. Future studies profiling individual FA reference ranges in many unique, global cohorts are necessary to develop cut-off values of individual FA concentrations highly related to disease-risk.
\end{abstract}

Keywords: fatty acids; lipids; omega-3; cholesterol; triglycerides; reference range; Singapore

\section{Introduction}

Adequate consumption of dietary fatty acids (FA) is essential for the development and maintenance of overall human health and well-being [1]. Due to their wide array of structural and functional capabilities, FA status has been implicated in reducing the risk of a number of chronic diseases [2,3]. There is currently a gap in the field of dietary lipid research as individual FA reference ranges have yet to be established. This fundamental gap hinders the capability of translating reported values from scientific reports for clinical use. Objective measures with established clinical ranges for other lipids such as cholesterol, and triglycerides are commonly used in clinical practices [4]. Developing baseline FA values would provide medical practitioners context regarding the direction and magnitude of change necessary to achieve optimal health in patients with deficient, suboptimal, or even excess amounts of FA. Additionally, FA values which are reported in scientific literature are often inconsistent, using either different units or methods of reporting altogether [5]. In order to address this gap, comprehensive profiles of baseline FA concentrations must be collected and analyzed. Developing validated FA reference ranges while using consistent 
methods of assessment and reporting are necessary steps in creating generalizable results between individual FA status of different cohorts.

To date, only a small number of studies have comprehensively profiled plasma or serum FA levels in healthy adults with the primary objective of developing reference ranges [6-9]. No studies to our knowledge have comprehensively examined FA status of individuals in the Singapore-Southeast Asia region of the world. Due to its unique geographical location as an island country located between Malaysia and Indonesia, Singapore has a unique population consisting of three predominant ethnic groups: Chinese, Malays, and Indian [10]. Independent of ethnicity, rates of obesity in Singapore are steadily increasing as their typical diets are very energy-dense [11]. A rise in western-style fast food and high rates of Singaporeans typically going out to eat, has been positively correlated with these dietary changes [11,12]. Additionally, the rise in large-scale production of palm oil, high in the saturated fatty acid (SFA) palmitic acid (PA) and the monounsaturated fatty acid (MUFA) oleic acid (OA), from Indonesia and Malaysia have contributed to high levels of consumption in the region [13]. A number of studies have found significant correlations between FA status and the risk of chronic disease specifically in Singapore Chinese adults [14-23]. On a global scale, countries in Southeast Asia have reported far higher omega-3 polyunsaturated fatty acids (PUFA) and SFA dietary consumption than North American countries [24]. This study sought to assess and determine the distribution of sixty-seven individual FA concentrations in healthy, adult Singaporean men and women. The unique variance of FA status and lipid biomarkers associated with overall health status and differences between males and females were examined. Given that certain diseasestates are known to alter FA status independent of dietary intake [25], using only healthy participants will reduce potential confounding between FA modifications associated with a disease-state or natural fluctuation due to daily living.

\section{Materials and Methods}

\subsection{Study Subjects}

Participants were originally recruited as described in Bi et al. [26]. In brief, 476 healthy male and female adult Singaporeans were recruited from the general public through advertisements placed around the National University of Singapore and the Clinical Nutrition Research Centre website from June 2014 to January 2017. All participants lived in Singapore for at least the past five years and were excluded if they had been diagnosed with any major disease, were pregnant, taking oral contraceptives or, who were breastfeeding. All participants were instructed to not consume alcohol and caffeine-containing drinks for $24 \mathrm{~h}$ prior and to fast prior to data collection. All procedures involving human participants were approved by the National Healthcare Group Domain Specific Review Board (NHG DSRB, Reference Number: 2013/00783), Singapore and the University of Guelph (Research Ethics Board Number: 18-10-049).

\subsection{Anthropometric Measures}

Participants reached the laboratory at 8:30 AM after $10 \mathrm{~h}$ of an overnight fast, Anthropometric measurements were obtained in a fasted state. Weight $(\mathrm{kg})$ in light clothing without footwear was measured to the nearest $0.1 \mathrm{~kg}$ by using an electrical scale and height $(\mathrm{cm})$ was measured using a stadiometer to the nearest millimeter (Seca 763 digital scale, Birmingham, UK). Body mass index (BMI) $\left(\mathrm{kg} / \mathrm{m}^{2}\right)$ was calculated using weight divided by the height squared. Waist circumference (WC) was taken at the smallest WC above the umbilicus or navel and below the xiphoid process. DEXA (QDR 4500A, fan-beam densitometer, Hologic, Waltham, MA, USA) was used for the measurement of fat mass (\%). To ensure accuracy of the measurement, all metal items were removed from the participants.

\subsection{Blood Samples}

Blood samples were collected as previously described in Bi et al. [27]. In brief, $10 \mathrm{~mL}$ of venous blood was collected into Vacutainers (Becton Dickinson Diagnostics, Franklin Lakes, 
NJ, USA). Blood samples were separated by centrifugation at $1500 \times \mathrm{g}$ for $10 \mathrm{~min}$ at $4{ }^{\circ} \mathrm{C}$ within $2 \mathrm{~h}$ of being drawn and aliquots were stored at $-80^{\circ} \mathrm{C}$ until analysis. Fasting lipid parameters including total cholesterol, high-density lipoprotein (HDL), and low-density lipoprotein (LDL) cholesterol were measured using the COBAS c311 (Roche)chemistry analyzer. Measurements were done in duplicate and readings were averaged.

\subsection{Gas Chromatography Protocol}

A modified Folch method was used for lipid extraction for serum samples which has been described in-detail previously [28]. In brief, samples were first thawed on ice, where $50 \mu \mathrm{L}$ of plasma or serum was pipetted into a $15 \mathrm{~mL}$ tube. Stock solution of 2:1 chloroform:methanol (v:v) with a $3.33 \mu \mathrm{g} / \mathrm{mL}$ of internal standard (C19:0) was prepared in which $3 \mathrm{~mL}$ of $\mathrm{CHCl} 3$ : $\mathrm{MeOH}$ was added to the tube and capped tightly with a phenolic PTFE liner. After a one minute of vortex, $550 \mu \mathrm{L}$ of $0.1 \mathrm{M} \mathrm{KCl}$ was added to each tube and briefly vortexed again for another 5-10 s. Next, samples were spun at $1460 \mathrm{rpm}$ for $10 \mathrm{~min}\left(\right.$ at $21^{\circ} \mathrm{C}$ ) to separate phases. The lower chloroform layer was transferred into a clean $15 \mathrm{~mL}$ glass tube and dried down under a gentle stream of nitrogen. After, $300 \mu \mathrm{L}$ of hexane and $1 \mathrm{~mL} \mathrm{14 \%} \mathrm{BF3-MeOH}$ was added and followed with a vortex of 5-10 s. Methylation was then completed at $100 \mathrm{oC}$ for $60 \mathrm{~min}$ in oven, where it was then cooled for $10 \mathrm{~min}$ at room temperature. Next, $1 \mathrm{~mL}$ of double-distilled water and $1 \mathrm{~mL}$ hexane was added to stop the methylation process. After a one-minute vortex, the sample was once again spun down at $1460 \mathrm{rpm}$ for $10 \mathrm{~min}\left(\right.$ at $21^{\circ} \mathrm{C}$ ) to separate phases. The top hexane layer was then extracted into a clean $2 \mathrm{~mL}$ GC vial, dried down under a gentle stream of $\mathrm{N} 2$ and then reconstituted in $400 \mu \mathrm{L}$ of hexane.

Total FA were quantified on an Agilent 7890-A gas chromatography with flame ionization detector and separated on a SP-2560 fused silica capillary column (100 m $\times$ $0.25 \mathrm{~mm} \times 0.2 \mathrm{um}$ ) (Sigma, Cat \# 24056). The splitless inlet (pressure of $19.5 \mathrm{psi}$ and a hydrogen flow of $13.7 \mathrm{~mL} / \mathrm{min}$ ) and detector was set at $250{ }^{\circ} \mathrm{C}$ (hydrogen flow of $30 \mathrm{~mL} / \mathrm{min}$, air flow of $450 \mathrm{~mL} / \mathrm{min}$ and nitrogen flow of $10 \mathrm{~mL} / \mathrm{min}$ ), where $1 \mathrm{uL}$ of sample was injected onto a splitless inlet set. The oven was initially set at $60^{\circ} \mathrm{C}$, where it was increased to $13^{\circ} \mathrm{C} / \mathrm{min}$ to $170{ }^{\circ} \mathrm{C}$ and was held for $4 \mathrm{~min}$, then followed $6.5^{\circ} \mathrm{C} / \mathrm{min}$ to $175^{\circ} \mathrm{C}$ with no hold to $2.6^{\circ} \mathrm{C} / \mathrm{min}$ to $185^{\circ} \mathrm{C}$ with no hold, to $1.3^{\circ} \mathrm{C} / \mathrm{min}$ to $190^{\circ} \mathrm{C}$ with no hold, to $13.0^{\circ} \mathrm{C} / \mathrm{min}$ to $240^{\circ} \mathrm{C}$ and was held for $13 \mathrm{~min}$. Total run time was $49.78 \mathrm{~min}$ per sample.

\subsection{Fatty Acid Analysis}

Peaks were identified and chromatographs were analyzed via OpenLab CDS EZChrom Edition 3.2.1 software. Sixty-seven total FA were included in the pool analyzed and a C19:0 internal standard was used to calculate FA concentrations $(\mu \mathrm{mol} / \mathrm{L})$ and percent composition (\%). Non-detectable (nd) values indicate FA below the detectable limit $(0.05 \mu \mathrm{g} / \mathrm{mL})$ of the Agilent 7890-A GC-FID.

\subsection{Statistical Analysis}

Statistical analysis was conducted using SAS University Edition (SAS Institute Inc.; Cary, NC, USA). The range, mean, and percentiles of the concentration of all sixty-seven FA and triglycerides (TG), LDL, HDL, and total cholesterol were profiled. Of the sixtyseven FA analyzed, ten were chosen for further analysis due to their implications to health and prominence in the diet. A Student $t$-test was used to determine statistical differences between male and female participants with results expressed as mean \pm standard deviation (SD). A multiple regression model including age, sex, BMI, and FA was conducted to assess the contributions of FA concentrations on TG, LDL, HDL, and total cholesterol. Results were reported as standardized parameter estimates/beta ( $\beta$ ) for FA, accounting for the unique variance of the single predictor in the model (FA) while holding the other variables constant. A $p$-value of $<0.05$ was considered statistically significant. 


\section{Results}

The general characteristics of the entire study population, including differences between males and females, are presented in Table 1. All participants self-reported to be healthy, disease-free, and on average recorded normal BMI values $\left(22.4 \mathrm{~kg} / \mathrm{m}^{2}\right)$, based on North American and Asian Standards. Significant differences were noted between sexes in measures that are commonly seen in other populations such as: BMI, WC, and fat \%. Females had significantly greater levels of HDL cholesterol but both groups had higher concentrations compared to normal values [29]. Both sexes exhibited moderately high concentrations of LDL cholesterol, total cholesterol, and fat \%, but TG were well below reported normal levels of $1.7 \mathrm{mmol} / \mathrm{L}$ [29].

Table 1. General characteristics of study population.

\begin{tabular}{ccccc}
\hline & Total Population & Males & Females & $p$-Value \\
\hline Population (\#) & 476 & 186 & 290 & $/$ \\
Age $(\mathrm{yrs})$ & $38.9 \pm 14.6$ & $38.41 \pm 14.6$ & $39.21 \pm 14.7$ & 0.5601 \\
BMI $\left(\mathrm{kg} / \mathrm{m}^{2}\right)$ & $22.4 \pm 3.4$ & $23.3 \pm 2.9$ & $21.8 \pm 3.6$ & $<0.0001 *$ \\
Waist Circumference (cm) & $73.9 \pm 9.2$ & $79.3 \pm 8.1$ & $70.5 \pm 8.2$ & $<0.0001 *$ \\
DEXA Fat (\%) & $31.0 \pm 7.7$ & $24.7 \pm 5.7$ & $35.1 \pm 5.8$ & $<0.0001 *$ \\
Glucose (mmol/L) & $4.55 \pm 0.49$ & $4.63 \pm 0.51$ & $4.50 \pm 0.46$ & $0.0032^{*}$ \\
HDL Cholesterol (mmol/L) & $1.68 \pm 0.43$ & $1.51 \pm 0.35$ & $1.79 \pm 0.44$ & $<0.0001 *$ \\
LDL Cholesterol (mmol/L) & $3.35 \pm 0.92$ & $3.41 \pm 0.93$ & $3.32 \pm 0.91$ & 0.2771 \\
Total Cholesterol (mmol/L) & $5.27 \pm 1.03$ & $5.21 \pm 0.99$ & $5.31 \pm 1.05$ & 0.2868 \\
Triglycerides (mmol/L) & $0.97 \pm 0.45$ & $1.01 \pm 0.44$ & $0.95 \pm 0.46$ & 0.1328 \\
Total Serum Fatty Acids $(\mu \mathrm{mol} / \mathrm{L})$ & $11,458 \pm 2478$ & $11,379 \pm 2422$ & $11,509 \pm 2515$ & 0.5775 \\
\hline
\end{tabular}

General characteristics of the study population were compared between males and females using a Student $t$-test. * Considered statistically significant at $p<0.05$. Data represented as mean \pm standard deviation. DEXA; Dual-energy X-ray absorptiometry scan.

Table 2 details the mean, range, and percentiles of sixty-seven FA concentrations, expressed as $\mu \mathrm{mol} / \mathrm{L}$ for 476 participants. Of this large pool, ten common FA which together can account for more than $90 \%$ of the total FA concentration, were selected to be analyzed further due to their prominence in the diet and health implications.

Table 2. Range, mean, and percentiles of fatty acid concentrations ( $\mu \mathrm{mol} / \mathrm{L})$ of serum total lipids.

\begin{tabular}{|c|c|c|c|c|c|c|c|c|c|c|c|}
\hline \multirow[b]{2}{*}{ Fatty Acid } & \multicolumn{4}{|c|}{ Range } & \multicolumn{7}{|c|}{ Percentile } \\
\hline & Min. & Mean & SD & Max. & 5 & 10 & 25 & 50 & 75 & 90 & 95 \\
\hline 12:0 (Lauric) & $\mathrm{t}$ & 10.9 & 12.4 & 160.0 & $\mathrm{t}$ & 3.5 & 5.2 & 7.9 & 13.2 & 20.9 & 29.0 \\
\hline 14:0 (Myristic) & 25.4 & 82.8 & 47.0 & 342.7 & 33.6 & 39.5 & 48.9 & 69.8 & 100.0 & 146.1 & 180.1 \\
\hline 15:0 (Pentadecanoic) & $\mathrm{t}$ & 39.2 & 28.2 & 207.7 & 9.6 & 11.9 & 16.7 & 26.4 & 61.9 & 77.4 & 87.8 \\
\hline 16:0 (Palmitic) & 1400.4 & 2667.5 & 625.1 & 6161.0 & 1809.8 & 1991.3 & 2256.2 & 2563.0 & 2967.9 & 3529.2 & 3908.3 \\
\hline 17:0 (Margaric) & $\mathrm{t}$ & 37.8 & 15.4 & 162.0 & 17.6 & 22.2 & 28.1 & 35.6 & 45.0 & 56.2 & 66.3 \\
\hline 18:0 (Stearic) & 415.2 & 745.2 & 155.5 & 1550.6 & 522.5 & 568.8 & 641.5 & 720.9 & 828.5 & 946.0 & 1021.8 \\
\hline 20:0 (Arachidic) & 1.8 & 20.1 & 5.3 & 50.3 & 12.8 & 14.0 & 16.6 & 19.5 & 22.8 & 26.4 & 29.8 \\
\hline $21: 0$ & 0.7 & 8.7 & 7.2 & 120.1 & 3.5 & 4.3 & 5.7 & 7.4 & 9.8 & 13.0 & 16.9 \\
\hline 22:0 (Behenic) & 11.0 & 45.2 & 11.6 & 91.9 & 28.6 & 31.1 & 37.5 & 44.5 & 52.0 & 59.1 & 65.5 \\
\hline $23: 0$ & $\mathrm{t}$ & 7.0 & 9.2 & 36.3 & $\mathrm{t}$ & $\mathrm{t}$ & $\mathrm{t}$ & $\mathrm{t}$ & 15.5 & 20.7 & 23.0 \\
\hline 24:0 (Lignoceric) & 5.0 & 42.7 & 11.2 & 94.1 & 26.2 & 29.4 & 34.9 & 41.7 & 50.2 & 56.0 & 61.7 \\
\hline $12: 1 \mathrm{c} 11$ & nd & nd & & nd & nd & nd & nd & nd & nd & nd & nd \\
\hline 14:1c9 (Myristoleic) & $\mathrm{t}$ & 10.4 & 9.2 & 46.6 & $\mathrm{t}$ & $\mathrm{t}$ & 2.8 & 9.1 & 17.1 & 22.3 & 25.9 \\
\hline $15: 1 \mathrm{c} 10$ & nd & nd & & nd & nd & nd & nd & nd & nd & nd & nd \\
\hline 16:1c9 (Palmitoleic) & 6.5 & 196.0 & 84.2 & 526.4 & 88.8 & 105.9 & 135.1 & 180.2 & 238.1 & 309.0 & 359.4 \\
\hline $17: 1 \mathrm{c} 10$ & nd & nd & & nd & nd & nd & nd & nd & nd & nd & nd \\
\hline 18:1c9 (Oleic) & 263.1 & 2095.7 & 633.1 & 5070.1 & 1311.1 & 1431.0 & 1683.2 & 1975.6 & 2389.2 & 2969.2 & 3381.2 \\
\hline 18:1c11 (cis-Vaccenic) & 13.1 & 163.9 & 43.2 & 355.9 & 106.5 & 115.7 & 134.7 & 159.5 & 186.7 & 218.8 & 240.3 \\
\hline 18:1c12 & $\mathrm{t}$ & 4.2 & 3.3 & 17.3 & $\mathrm{t}$ & $\mathrm{t}$ & 2.1 & 3.9 & 5.8 & 8.1 & 10.8 \\
\hline 18:1c13 & $\mathrm{t}$ & $\mathrm{t}$ & & $\mathrm{t}$ & $\mathrm{t}$ & $\mathrm{t}$ & $\mathrm{t}$ & $\mathrm{t}$ & $\mathrm{t}$ & $\mathrm{t}$ & $\mathrm{t}$ \\
\hline 18:1c14 & $\mathrm{t}$ & $\mathrm{t}$ & & $\mathrm{t}$ & $\mathrm{t}$ & $\mathrm{t}$ & $t$ & $\mathrm{t}$ & $\mathrm{t}$ & $\mathrm{t}$ & $\mathrm{t}$ \\
\hline 19:1c10 & nd & nd & & nd & nd & nd & nd & nd & nd & nd & nd \\
\hline $20: 1 c 5$ & $t$ & 6.3 & 2.9 & 18.8 & 3.0 & 3.3 & 4.3 & 5.6 & 7.4 & 10.7 & 12.2 \\
\hline $20: 1 c 8$ & $\mathrm{t}$ & 3.0 & 1.6 & 18.0 & 1.2 & 1.5 & 2.0 & 2.7 & 3.7 & 4.8 & 5.9 \\
\hline
\end{tabular}


Table 2. Cont.

\begin{tabular}{|c|c|c|c|c|c|c|c|c|c|c|c|}
\hline \multirow[b]{2}{*}{ Fatty Acid } & \multicolumn{4}{|c|}{ Range } & \multicolumn{7}{|c|}{ Percentile } \\
\hline & Min. & Mean & SD & Max. & 5 & 10 & 25 & 50 & 75 & 90 & 95 \\
\hline 20:1c11 (Gondoic) & 2.6 & 16.2 & 5.5 & 52.5 & 9.8 & 10.8 & 12.7 & 15.4 & 18.5 & 23.0 & 26.5 \\
\hline 22:1n9 (Erucic) & $\mathrm{t}$ & 4.2 & 4.8 & 74.3 & $\mathrm{t}$ & 1.9 & 2.4 & 3.3 & 5.2 & 7.9 & 8.9 \\
\hline 24:1n9 (Nervonic) & 2.6 & 63.9 & 17.7 & 187.1 & 41.1 & 44.1 & 52.7 & 61.6 & 72.7 & 84.2 & 92.3 \\
\hline 16:1t9 (Palmitelaidic) & $\mathrm{t}$ & 7.9 & 5.7 & 27.1 & 2.0 & 2.2 & 2.9 & 6.8 & 10.9 & 16.3 & 19.9 \\
\hline $18: 1 \mathrm{t} 4$ & $\mathrm{t}$ & 0.2 & 1.4 & 26.8 & $\mathrm{t}$ & $\mathrm{t}$ & $\mathrm{t}$ & $\mathrm{t}$ & $\mathrm{t}$ & $\mathrm{t}$ & 1.1 \\
\hline $18: 1 \mathrm{t} 5$ & $\mathrm{t}$ & 6.6 & 8.8 & 62.2 & $\mathrm{t}$ & $\mathrm{t}$ & $\mathrm{t}$ & 3.6 & 9.1 & 17.9 & 25.0 \\
\hline $18: 1 \mathrm{t} 6-8$ & $\mathrm{t}$ & 3.3 & 2.5 & 16.5 & 0.7 & 0.9 & 1.5 & 2.7 & 4.6 & 6.5 & 8.4 \\
\hline 18:1t9 (Elaidic) & $\mathrm{t}$ & 6.7 & 3.3 & 47.0 & 2.7 & 3.5 & 4.7 & 6.3 & 8.1 & 10.0 & 11.9 \\
\hline $18: 1 \mathrm{t} 10$ & $\mathrm{t}$ & 4.4 & 3.1 & 29.7 & 1.1 & 1.8 & 2.6 & 3.6 & 5.4 & 7.5 & 9.4 \\
\hline 18:1t11(trans-Vaccenic) & $\mathrm{t}$ & 7.1 & 5.0 & 59.4 & 1.7 & 2.4 & 3.9 & 6.0 & 8.9 & 12.1 & 15.9 \\
\hline $18: 1 \mathrm{t} 12$ & $\mathrm{t}$ & 4.4 & 3.8 & 59.9 & 1.5 & 1.9 & 2.7 & 3.6 & 5.1 & 7.8 & 9.8 \\
\hline $18: 1 \mathrm{t} 13$ & $\mathrm{t}$ & 11.0 & 7.0 & 59.3 & 4.3 & 5.1 & 6.9 & 9.4 & 13.2 & 18.0 & 21.9 \\
\hline $18: 1 \mathrm{t} 16$ & $\mathrm{t}$ & 3.6 & 2.4 & 26.0 & $\mathrm{t}$ & $\mathrm{t}$ & 2.4 & 3.4 & 4.6 & 6.4 & 7.5 \\
\hline $18: 2 \mathrm{tt}$ & $\mathrm{t}$ & 4.1 & 4.4 & 25.9 & $\mathrm{t}$ & $\mathrm{t}$ & 0.9 & 2.7 & 5.5 & 10.4 & 13.6 \\
\hline 18:2t9t12 (Linoelaidic) & $\mathrm{t}$ & 1.2 & 3.0 & 25.6 & $\mathrm{t}$ & $\mathrm{t}$ & $\mathrm{t}$ & $\mathrm{t}$ & $\mathrm{t}$ & 4.6 & 6.7 \\
\hline $18: 2 \mathrm{c} 9 \mathrm{t} 13$ & $\mathrm{t}$ & 1.1 & 2.6 & 17.8 & $\mathrm{t}$ & $\mathrm{t}$ & $\mathrm{t}$ & $\mathrm{t}$ & $\mathrm{t}$ & 4.9 & 7.0 \\
\hline $18: 2 \mathrm{ct}$ & $\mathrm{t}$ & 5.2 & 3.2 & 24.1 & 2.0 & 2.5 & 3.3 & 4.4 & 6.0 & 8.8 & 11.7 \\
\hline $18: 2 \mathrm{c} 9 \mathrm{t} 12$ & 1.0 & 13.7 & 4.6 & 38.5 & 8.1 & 9.4 & 10.8 & 12.6 & 15.6 & 20.0 & 23.2 \\
\hline $18: 2 \mathrm{t} 9 \mathrm{c} 12$ & $\mathrm{t}$ & 7.9 & 3.2 & 21.0 & 3.2 & 4.2 & 5.8 & 7.7 & 9.5 & 11.9 & 13.4 \\
\hline $18: 2 c 9 c 14$ & $\mathrm{t}$ & 1.3 & 2.4 & 18.2 & $\mathrm{t}$ & $\mathrm{t}$ & $\mathrm{t}$ & $\mathrm{t}$ & 2.1 & 3.9 & 6.3 \\
\hline 18:2c9c15 (Mangiferic) & $\mathrm{t}$ & 2.5 & 3.6 & 21.3 & $\mathrm{t}$ & $\mathrm{t}$ & $\mathrm{t}$ & $\mathrm{t}$ & 4.5 & 7.2 & 9.2 \\
\hline 18:2c9t11 (Rumenic) & $\mathrm{t}$ & 8.7 & 2.6 & 23.4 & 4.8 & 6.2 & 7.4 & 8.5 & 9.7 & 11.5 & 13.1 \\
\hline 18:2c11t13 (CLA) & $\mathrm{t}$ & 1.0 & 2.0 & 10.7 & $\mathrm{t}$ & $\mathrm{t}$ & $\mathrm{t}$ & $\mathrm{t}$ & $\mathrm{t}$ & 4.3 & 5.9 \\
\hline 18:2t10c12 (CLA) & $\mathrm{t}$ & 0.6 & 1.8 & 13.0 & $\mathrm{t}$ & $\mathrm{t}$ & $\mathrm{t}$ & $\mathrm{t}$ & $\mathrm{t}$ & 2.6 & 5.1 \\
\hline $18: 2 c / c$ isomer (CLA) & $\mathrm{t}$ & 0.3 & 1.2 & 12.7 & $\mathrm{t}$ & $\mathrm{t}$ & $\mathrm{t}$ & $\mathrm{t}$ & $\mathrm{t}$ & $\mathrm{t}$ & 1.7 \\
\hline $18: 2 c / c$ isomer (CLA) & $\mathrm{t}$ & 2.1 & 2.4 & 16.2 & $\mathrm{t}$ & $\mathrm{t}$ & $\mathrm{t}$ & 1.7 & 3.0 & 4.7 & 6.6 \\
\hline $18: 2 \mathrm{tt}(\mathrm{CLA})$ & $\mathrm{t}$ & 8.9 & 4.4 & 38.4 & 0.6 & 4.7 & 6.8 & 8.6 & 10.7 & 13.1 & 15.2 \\
\hline 18:2n6 (Linoleic acid) & 1652.5 & 3700.2 & 777.5 & 7244.2 & 2559.2 & 2766.6 & 3181.0 & 3640.6 & 4090.3 & 4771.7 & 5081.2 \\
\hline 18:3n6 ( $\gamma$-linolenic) & 2.9 & 27.0 & 19.7 & 124.5 & 7.5 & 8.7 & 12.7 & 22.5 & 35.0 & 51.6 & 63.2 \\
\hline 20:2n6 (Eicosadienoic) & 2.5 & 24.0 & 7.8 & 52.7 & 12.6 & 15.8 & 18.8 & 22.8 & 28.4 & 33.6 & 38.8 \\
\hline 20:3n6 (Dihomo- $\gamma$-linolenic) & 2.0 & 115.3 & 46.3 & 273.9 & 56.6 & 64.0 & 80.1 & 106.4 & 141.4 & 181.1 & 206.4 \\
\hline 20:4n6 (Arachidonic) & 104.3 & 685.8 & 177.4 & 1538.7 & 433.7 & 480.0 & 573.0 & 673.9 & 784.6 & 908.3 & 973.5 \\
\hline 22:2n6 (Docosadienoic) & $\mathrm{t}$ & 7.9 & 4.8 & 33.5 & 2.5 & 3.2 & 4.6 & 6.5 & 10.3 & 14.3 & 16.7 \\
\hline 22:4n6 (Adrenic) & 3.6 & 17.7 & 5.9 & 50.4 & 9.3 & 11.1 & 14.1 & 17.2 & 20.9 & 25.2 & 27.4 \\
\hline 22:5n6 (Docosapentaenoic) & 3.7 & 19.7 & 6.2 & 44.7 & 11.0 & 12.6 & 15.4 & 19.0 & 23.4 & 27.5 & 31.1 \\
\hline 20:3n9 (Mead) & 3.0 & 8.9 & 4.1 & 29.4 & 4.3 & 4.9 & 6.2 & 8.1 & 10.2 & 13.9 & 17.1 \\
\hline 18:3n3 ( $\alpha$-linolenic) & 7.3 & 57.6 & 24.7 & 175.4 & 28.7 & 33.0 & 41.1 & 52.4 & 69.7 & 91.6 & 103.3 \\
\hline 18:4n3 (Stearidonic) & $\mathrm{t}$ & $\mathrm{t}$ & & $\mathrm{t}$ & $\mathrm{t}$ & $\mathrm{t}$ & $\mathrm{t}$ & $\mathrm{t}$ & $\mathrm{t}$ & $\mathrm{t}$ & $\mathrm{t}$ \\
\hline 20:3n3 (Dihomolinoleic) & $\mathrm{t}$ & 16.6 & 11.3 & 47.1 & $\mathrm{t}$ & 2.3 & 4.5 & 19.2 & 26.2 & 30.9 & 33.8 \\
\hline 20:5n3 (Eicosapentaenoic) & 4.2 & 93.0 & 80.9 & 596.3 & 20.1 & 29.0 & 40.0 & 68.2 & 115.2 & 193.42 & 285.1 \\
\hline 22:3n3 (Docosatrienoic Acid) & nd & nd & & nd & nd & nd & nd & nd & nd & nd & nd \\
\hline 22:5n3 (Docosapentaenoic) & 14.7 & 43.5 & 15.5 & 118.7 & 23.7 & $27 / 0$ & 32.5 & 40.7 & 50.5 & 65.3 & 71.5 \\
\hline 22:6n3 (Docosahexaenoic) & 44.9 & 255.5 & 94.4 & 663.4 & 121.9 & 150.7 & 193.56 & 240.5 & 304.0 & 391.4 & 430.1 \\
\hline
\end{tabular}

Reference ranges of sixty-seven fatty acids. 19:0 was used as an internal standard and not included in the table. CLA; conjugated linoleic acid t; Trace values recorded. nd; Non-detectable levels.

In Table 3, significant differences in the concentrations of SA, PAO, ALA, and DHA $(p<0.05)$ were found between males and females. Females demonstrated higher mean FA concentrations in all four FA which reached significance. 
Table 3. Fatty acids concentrations in males and females.

\begin{tabular}{ccccc}
\hline Fatty Acid & Common Name & $\begin{array}{c}\text { Males } \\
(\mathbf{n}=\mathbf{1 8 6})\end{array}$ & $\begin{array}{c}\text { Females } \\
(\mathbf{n}=\mathbf{2 9 0})\end{array}$ & $p$-Value \\
\hline $16: 0$ & Palmitic acid (PA) & $2683 \pm 630.6$ & $2658 \pm 622.4$ & 0.66 \\
$18: 0$ & Stearic acid (SA) & $716 \pm 145.2$ & $764 \pm 159.2$ & $0.001^{*}$ \\
$16: 1 \mathrm{c} 9$ & Palmitoleic acid (PAO) & $185 \pm 82.5$ & $203 \pm 84.7$ & $0.03^{*}$ \\
$18: 1 \mathrm{c} 9$ & Oleic acid (OA) & $2117 \pm 645.0$ & $2082 \pm 625.9$ & 0.55 \\
$18: 2 \mathrm{n} 6$ & Linoleic acid (LA) & $3652 \pm 737.4$ & $3731 \pm 801.9$ & 0.28 \\
$20: 4 \mathrm{n} 6$ & Arachidonic acid (ARA) & $687 \pm 176.2$ & $685 \pm 178.5$ & 0.86 \\
$18: 3 \mathrm{n} 3$ & Alpha-linolenic acid (ALA) & $53.9 \pm 23.0$ & $60.0 \pm 25.4$ & $0.008^{*}$ \\
$20: 5 \mathrm{n} 3$ & Eicosapentaenoic acid (EPA) & $84.4 \pm 74.2$ & $98.5 \pm 84.7$ & 0.06 \\
$22: 5 \mathrm{n} 3$ & Docosapentaenoic acid (DPA) & $42.6 \pm 14.8$ & $44.1 \pm 0.9$ & 0.3 \\
$22: 6 n 3$ & Docosahexaenoic acid (DHA) & $240 \pm 86.8$ & $266 \pm 97.8$ & $0.003^{*}$ \\
\hline
\end{tabular}

Fatty acid concentrations of ten biologically relevant fatty acids are compared between male and female participants by the Student's $t$-test. Results are presented as mean \pm standard deviation. * Considered statistically significant at $p<0.05$.

The mean, range, and percentiles of TG, HDL, LDL, and total cholesterol for the entire study population of 476 participants is profiled in Table 4 . The cut-off values for lipid biomarkers used by the Singapore Ministry of Health which denotes a health risk are also included [29]. A majority of participants exhibited desirable levels of TG and HDL and borderline/high levels of LDL and total cholesterol (TC).

Table 4. Reference ranges and clinical cut-offs of lipid biomarkers.

\begin{tabular}{|c|c|c|c|c|c|c|c|c|c|c|}
\hline \multirow[b]{2}{*}{ Class } & \multicolumn{3}{|c|}{ Range } & \multicolumn{7}{|c|}{ Percentile } \\
\hline & Min & Mean \pm SD & $\operatorname{Max}$ & 5 & 10 & 25 & 50 & 75 & 90 & 95 \\
\hline TG & 0.36 & $0.97 \pm 0.45$ & 3.06 & 0.50 & 0.55 & 0.66 & 0.87 & 1.13 & 1.64 & 1.91 \\
\hline HDL & 0.80 & $1.68 \pm 0.43$ & 3.44 & 1.07 & 1.16 & 1.38 & 1.65 & 1.91 & 2.29 & 2.48 \\
\hline LDL & 0.99 & $3.35 \pm 0.92$ & 7.64 & 2.06 & 2.29 & 2.74 & 3.23 & 3.87 & 4.48 & 4.99 \\
\hline $\mathrm{TC}$ & 2.70 & $5.27 \pm 1.03$ & 9.44 & 3.74 & 4.08 & 4.58 & 5.17 & 5.85 & 6.67 & 7.05 \\
\hline Class & \multicolumn{2}{|c|}{ Desirable $^{a}$} & \multicolumn{4}{|c|}{ Borderline/High } & \multicolumn{4}{|c|}{ Very High } \\
\hline TG & \multirow{2}{*}{\multicolumn{2}{|c|}{$\begin{array}{l}1.7-2.2 \mathrm{mmol} / \mathrm{L} \\
1.0-1.5 \mathrm{mmol} / \mathrm{L}\end{array}$}} & \multicolumn{4}{|c|}{$2.3-4.4 \mathrm{mmol} / \mathrm{L}$} & \multicolumn{4}{|c|}{$\geq 4.5 \mathrm{mmol} / \mathrm{L}$} \\
\hline HDL & & & \multicolumn{4}{|c|}{$>1.6 \mathrm{mmol} / \mathrm{L}$} & & & & \\
\hline LDL & \multicolumn{2}{|c|}{$2.6-3.3 \mathrm{mmol} / \mathrm{L}$} & \multirow{2}{*}{\multicolumn{4}{|c|}{$\begin{array}{l}3.4-4.8 \mathrm{mmol} / \mathrm{L} \\
5.2-6.1 \mathrm{mmol} / \mathrm{L}\end{array}$}} & \multicolumn{4}{|c|}{$\geq 4.9 \mathrm{mmol} / \mathrm{L}$} \\
\hline $\mathrm{TC}$ & \multicolumn{2}{|c|}{$<5.2 \mathrm{mmol} / \mathrm{L}$} & & & & & & \multicolumn{2}{|c|}{$\geq 6.2 \mathrm{mmol} / \mathrm{L}$} & \\
\hline
\end{tabular}

The top half of the table displays the range of values for the four lipid biomarkers evaluated for all participants in this cohort. The bottom half of the table details the clinical cut-off values associated with chronic health risk of each of the four lipid biomarkers as classified by Ministry of Health for Singapore. a Tai et al. [29]. HDL; high-density lipoprotein, LDL; low-density lipoprotein, TC; total cholesterol, TG; triglycerides.

In Table 5, the variance accounted for by FA concentrations, when holding age, sex and BMI constant, and lipid biomarkers were significant for almost all cholesterol and TG values. Only EPA did not exhibit a significant result with TG $(\beta=0.00)$. All ten FA significantly contributed to LDL and total cholesterol. A majority of the FA exhibited significant and positive contributions to HDL cholesterol, but PA, PAO, OA, and ALA did not reach statistical significance. Total FA concentrations all reached significance with circulating triglycerides, HDL, LDL, and total cholesterol. 
Table 5. Multiple regression model assessing the unique variance between select fatty acids and lipid biomarkers.

\begin{tabular}{|c|c|c|c|c|c|}
\hline Fatty Acid & Common Name & Triglycerides & HDL & LDL & Total Cholesterol \\
\hline & & $\beta$ & $\beta$ & $\beta$ & $\beta$ \\
\hline $16: 0$ & Palmitic acid (PA) & $0.78 *$ & 0.02 & $0.55 *$ & 0.64 * \\
\hline 18:0 & Stearic acid (SA) & $0.64 *$ & $0.18^{*}$ & $0.60 *$ & $0.72 *$ \\
\hline $16: 1 c 9$ & Palmitoleic acid (PAO) & $0.70 *$ & -0.05 & $0.31 *$ & $0.38 *$ \\
\hline $18: 1 c 9$ & Oleic acid (OA) & $0.81 *$ & -0.10 & $0.46^{*}$ & $0.50 *$ \\
\hline $18: 2 \mathrm{n} 6$ & Linoleic acid (LA) & $0.45 *$ & $0.24 *$ & $0.62 *$ & $0.72 *$ \\
\hline $20: 4 n 6$ & Arachidonic acid (ARA) & $0.17 *$ & $0.25 *$ & $0.43 *$ & $0.37^{*}$ \\
\hline $18: 3 n 3$ & Alpha-linolenic acid (ALA) & $0.61 *$ & -0.06 & $0.29 *$ & $0.34 *$ \\
\hline $20: 5 \mathrm{n} 3$ & Eicosapentaenoic acid (EPA) & 0 & $0.20 *$ & $0.22 *$ & $0.29 *$ \\
\hline $22: 5 \mathrm{n} 3$ & Docosapentaenoic acid (DPA) & 0.33 * & $0.19 *$ & $0.40 *$ & $0.49 *$ \\
\hline \multirow[t]{2}{*}{$22: 6 n 3$} & Docosahexaenoic acid (DHA) & $0.27 *$ & $0.21 *$ & $0.44 *$ & $0.52 *$ \\
\hline & TOTAL & $0.67 *$ & $0.12 * *$ & $0.59 *$ & $0.69 *$ \\
\hline
\end{tabular}

A multiple regression model was conducted to examine the relationship between ten biologically relevant fatty acids and the four lipid biomarkers measured. The model included the fatty acid along with age, sex, and BMI. The $\beta$ reported is for the relationship between the fatty acid of interest and the lipid biomarker. ${ }^{*} p<0.001,{ }^{* *} p<0.05$. HDL; high-density lipoprotein, LDL; low-density lipoprotein.

Based on the cut-off values described in Table 4, the number of participants who exhibited levels of TG, HDL, LDL, or TC which exceeded recommended values are tallied in Table 6. Using the reference ranges of this cohort described in Table 2, FA concentrations in the $\geq 95$ th or $\geq 75$ th percentile and $\leq 25$ th or $\leq 5$ th percentile, were evaluated. In the 11 participants with high TG values, a majority of participants were also found to have SFA and MUFA concentrations in the $\geq 95$ th percentile, but very few or none had n-3 or n-6 PUFA exceeding the 95th percentile. There were 11 participants with low HDL cholesterol values and a few also had fatty acid concentrations exceeding the 75th percentile or below the 25th percentile. LDL and TC exhibited similar trends, with a large number of participants with FA concentrations in the $\geq 75$ th percentile across all ten FA and exceeding LDL and TC cutoffs. Overall, FA concentrations in the $\geq 95$ th percentile were far more prevalent in participants exceeding lipid cut-off values than concentrations in the $\leq 5$ th percentile.

Table 6. Exploratory investigation of the upper and lower percentile fatty acid concentrations in participants who exceed risk cut-offs for lipid biomarkers.

\begin{tabular}{|c|c|c|c|c|c|c|c|c|c|c|c|c|}
\hline Lipid Class & $\begin{array}{c}\text { Exceeded } \\
\text { Cut-Off }^{\text {a }}(\#)\end{array}$ & Percentile & $16: 0$ & $18: 0$ & $16: 1 c 9$ & 18:1c9 & $18: 2 n 6$ & $20: 4 n 6$ & $18: 3 n 3$ & $20: 5 n 3$ & $22: 5 n 3$ & $22: 6 n 3$ \\
\hline \multirow{2}{*}{ TG } & \multirow{2}{*}{11} & $\geq 95 \% \mathrm{~b}$ & 10 & 8 & 7 & 11 & 3 & 0 & 6 & 0 & 1 & 1 \\
\hline & & $\leq 5 \%$ b & 0 & 0 & 0 & 0 & 0 & 0 & 0 & 0 & 0 & 0 \\
\hline \multirow{2}{*}{ HDL-Low } & \multirow{2}{*}{11} & $\geq 75 \%{ }^{c}$ & 4 & 3 & 2 & 4 & 1 & 0 & 3 & 2 & 2 & 2 \\
\hline & & $\leq 25 \%$ c & 3 & 5 & 3 & 1 & 4 & 5 & 4 & 4 & 6 & 4 \\
\hline \multirow{2}{*}{ LDL } & \multirow{2}{*}{91} & $\geq 75 \%{ }^{c}$ & 62 & 63 & 38 & 54 & 65 & 46 & 44 & 39 & 52 & 52 \\
\hline & & $\leq 25 \%{ }^{c}$ & 1 & 1 & 6 & 0 & 1 & 9 & 5 & 9 & 5 & 5 \\
\hline \multirow{2}{*}{$\mathrm{TC}$} & \multirow{2}{*}{82} & $\geq 75 \%{ }^{c}$ & 54 & 60 & 37 & 50 & 66 & 52 & 43 & 43 & 53 & 52 \\
\hline & & $\leq 25 \%{ }^{c}$ & 0 & 0 & 5 & 1 & 0 & 7 & 4 & 7 & 2 & 4 \\
\hline
\end{tabular}

Values represent number of participants who exceed the risk cut-off values for lipid biomarkers and are also in the upper/lower 5th and 25th percentile of fatty acids. These percentiles were chosen to best capture the totality of participants who exceeded biomarker cut-off values. ${ }^{a}$ TG $\geq 2.3 \mathrm{mmol} / \mathrm{L} ; \mathrm{HDL}-\mathrm{Low}<1.0 \mathrm{mmol} / \mathrm{L} ; \mathrm{LDL} \geq 4.1 \mathrm{mmol} / \mathrm{L} ; \mathrm{TC} \geq 6.2 \mathrm{mmol} / \mathrm{L}$. ${ }^{\mathrm{b}}$ Each fatty acid has 24 total participants in each of the upper and lower 5 th percentile. ${ }^{c}$ Each fatty acid has 119 total participants in each of the upper and lower 25th percentile, except 18:3n3 which has 120. HDL; high-density lipoprotein, LDL; low-density lipoprotein, TC; total cholesterol, TG; triglycerides.

\section{Discussion}

In this study we determined the percentiles, mean, and ranges of sixty-seven FA collected from 476 healthy, adult Singaporean males and females with ten FA highlighted for further analysis with TG and cholesterol. Significant results between FA concentrations and TG, LDL, HDL, and total cholesterol were seen in almost all of the ten FA highlighted. Mean total FA concentrations reached significance with all four lipid biomarkers but exhibited 
the weakest relationship with HDL cholesterol. Individual FA concentrations were found to have a significant relationship to all four lipid biomarkers and high concentrations of FA were more often seen in individuals exceeding established cut-off values. Female participants exhibited significantly higher concentrations SA, PAO, ALA, and DHA when compared to males (Table 3), but total FA concentrations did not statistically differ between sexes (Table 1). To our knowledge, the inclusion of sixty-seven FA concentrations is the largest pool of FA to be examined to date for the purposes of profiling FA reference ranges in any cohort.

\subsection{Individual Fatty Acid Reference Ranges}

A very small number of studies have, in detail, profiled FA concentrations in healthy adults for the purposes of developing reference ranges [6-9]. Glew et al. [30] have also reported mean percent composition of twenty-six FA from serum samples collected in healthy adult males and females from Northern Nigeria. Of these studies, Bradbury et al. [9] and Glew et al. [30] reported their values in percent composition, whereas absolute concentrations were reported in our study. Sergeant et al. [7] $(n=93$; obese American women) and Abdelmagid [6] ( $\mathrm{n}=826$; healthy, multiethnic Canadians) also reported their results as absolute concentrations $(\mu \mathrm{mol} / \mathrm{L})$ which allows for the most direct and reliable comparisons to our results. Percent composition is determined by the percentage of one FA from the total pool of FA included in the analysis; a smaller pool analyzed results in a larger apparent percentage of FA [5]. Large fluctuations in a single FA could significantly modify the total value of the pool and subsequent percent composition seen in other FA when no changes in concentration actually occurred. Absolute concentrations of an FA are independent of changes in the pool analyzed and have a larger dynamic range of values, as percent is limited to a $0-100$ scale. Reporting results as absolute concentrations has been found to reduce the loss of statistical significance between FA compared to when values were assessed as weight percentages [31]. Furthermore, relative percent can be derived from absolute concentrations, but the same is not possible in reverse as the values of the internal standard used in the study are needed to calculate absolute concentration [5]. As our study included over sixty FA, whereas Bradbury et al. [9] and Glew et al. [30] only included up to twenty-six, it is difficult to accurately compare our reported values even in terms of percent composition. Thus, the use of absolute concentrations is recommended for future studies profiling FA reference ranges to improve comparability and generalizability of results.

Three of the five studies mentioned previously have examined adult North American cohorts and the remaining studies examined cohorts from New Zealand and Nigeria [6-9,30]. To our understanding, ours is the first study to examine FA concentrations of a large cohort of Southeast Asian, specifically Singaporean, adults in this level of detail. Previous work done in a multiethnic Singaporean cohort found plasma FA composition is a strong, reliable biomarker reflecting dietary consumption in epidemiological studies [32]. The mean FA concentration in our study $(11,458 \mu \mathrm{mol} / \mathrm{L})$ more closely resembled that of Sergeant et al. [7], as Abdelmagid [6] reported a mean concentration which was $\sim 40 \%$ lower $(6948 \mu \mathrm{mol} / \mathrm{L})$. Of the ten FA highlighted in Table 3; SA, PAO, AA, and ALA concentrations were noticeably lower in our study whereas EPA and DHA were higher compared to Sergeant et al. [7]. A study conducted in NA by Asselin et al. [33] found a cohort of overweight, older participants $(\mathrm{BMI}=25.9$; age $=59)$ reported mean plasma FA concentrations $(15,169 \mu \mathrm{mol} / \mathrm{L})$ similar to that of Sergeant et al. [7] and over double that of the cohort in Abdelmagid et al. [6]. The primary difference that may account for the high concentrations reported in Sergeant et al. [7] is their use of obese middle-aged women (BMI = 33.3; age $=56.9)$ compared to healthy, young university students $(\mathrm{BMI}=22.8$; age $=22.6)$ in Abdelmagid et al. [6]. FA concentrations have been found to strongly correlate with increases in BMI, as obese individuals exhibited modified FA status compared to healthy controls [34-36]. Furthermore, as one ages, genetic adaptations occur which can modify FA status, primarily omega-3 PUFA [37]. Taken together, the FA concentrations reported 
in Sergeant et al. [7] may be more reflective of the average FA status of North Americans due to the high prevalence of obesity in recent years. The use of healthy university-aged students in Abdelmagid et al. [6] could reflect FA concentrations lower than what is expected of the average NA and is representative of a cohort that has reduced risk factors of chronic disease.

\subsection{Anthropometric Measures}

The high total FA concentrations seen in the present study are in contrast to the lower total FA reported in Abdelmagid et al. [6], but the BMI are nearly identical to that of the Singaporean cohort. Our cohort, although reporting a standard healthy BMI, WC, and fasting blood glucose, had mean FA concentrations similar to that of the obese group profiled in Sergeant et al. [7]. Cultural and dietary differences are important modifying factors affecting FA concentrations. First, SFA intake is greater in Singapore than that of NA [24]. The countries surrounding Singapore are the largest producers of palm oil on the planet, which is high in PA, OA, and LA [13]. When examining the mean difference between PA, OA, and LA concentrations between our Singaporean cohort and the Canadian cohort of Abdelmagid et al. [6], those three FA alone account for $\sim 75 \%$ of the difference in mean total FA concentrations (calculation not shown). Due to the abundance of palm oil produced in Southeast Asia, dietary intakes have increased which have also been associated with a rise in CVD-related deaths [38]. Palm oil has also been found to strongly correlate with increased LDL cholesterol levels [38], which were reported to be borderline high in our study [6]. Similarly, frequency of omega-3 PUFA consumption via fish is low in NA, but consumption in Southeast Asia are some of the highest in the world [24,39]. Nearly $34.3 \%$ of adults in Singapore are overweight or obese and $33.1 \%$ are not meeting daily physical activity requirements as of $2014[40,41]$. Measures of body fat \% in both males and females in the present study were also quite high compared to other Asian cohorts [42]. In Caucasian men and women, an obese BMI classification of $30 \mathrm{~kg} / \mathrm{m}^{2}$ corresponds to approximately $25 \%$ and $35 \%$ body fat [43], equating to roughly the same body fat \% reported of both males and females in this study (Table 1). This phenomenon of a low BMI, but high body fat \% in Singaporean adults has also been described in previous studies [44]. Widely reported differences between Caucasian populations and Asian populations have resulted in the WHO to make amendments to the current BMI cut-offs for Asian cohorts which was adopted by Singapore in $2005[45,46]$. Current WHO BMI cut points for being considered overweight is $23-27.5 \mathrm{~kg} / \mathrm{m}^{2}$ and obese is $\geq 27.5 \mathrm{~kg} / \mathrm{m}^{2}$ for Asian populations [46]. Although the participants included in our study exhibited healthy levels of TG and HDL cholesterol, and were screened for any chronic diseases, their BMI and body fat \% should not be considered to be optimal. Due to this unique paradox found in the Singaporean population, interpretations between FA concentrations and health outcomes related to BMI in other reports should be compared cautiously. Thus, the high FA concentrations seen in our study can be interpreted as a risk factor to chronic disease, similar to that seen in other populations with higher reported body fat \% and BMI.

\subsection{Relationship with Lipid Biomarkers}

Unlike FA concentrations, established clinical reference ranges for TG and cholesterol have been established and are routinely utilized in clinical practices to assess health outcomes [4]. By evaluating the relationship between FA concentrations and lipid biomarkers, individual FA can be targeted to determine levels which may be deemed adequate or in excess. Mean LDL and total cholesterol levels in this study were found to be borderline high, which is associated with CVD disease risk $(3.35 \pm 0.92 ; 5.27 \pm 1.03)$ [29]. HDL levels were classified as being high $(1.68 \pm 0.43)$, which has shown to promote cardioprotective effects [29]. In Table 5, only PA, PAO, OA, and ALA did not reach statistical significance with HDL cholesterol, which has also been seen in previous studies [47-49]. EPA and DHA were found to significantly and positively contribute in our model with HDL cholesterol, similar to many previous studies citing strong positive correlations between omega-3 PUFA 
consumption and the antiatherogenic properties of HDL cholesterol levels [50]. Results of our study support the strong positive correlations between PA and SA to LDL and total cholesterol levels reported previously; a risk factor for developing CHD [51]. Compared to other common vegetable cooking oils low in SFA, palm oil, which is widely consumed in Singapore, has been found to increase LDL cholesterol levels significantly and subsequently increasing the risk of developing type II diabetes [38]. Thus, the values reported in Table 5 for the SFA, PA, and SA, could be disproportionately high based on the dietary habits of Singaporean adults. Unfortunately, dietary data was not available for this study.

The cohort of Singaporeans in the current study had generally very healthy TG levels, with only participants in the 95th percentile and up reaching borderline high values (based on Asian cutoffs) and none of our participants were classified as having very high TG levels (Table 4) [29,52]. Results in Table 5 between PA, SA, PAO, OA, and TG concentrations are similar to the findings reported in previous studies $[6,53,54]$. High levels of TG have been found to increase the risk of developing CVD, atherosclerosis, and are often seen in obese individuals and those with type- 2 diabetes $[55,56]$. Southeast-Asians on average consume some of the highest levels of omega-3 PUFA via fish in the world, which is reflected by the concentrations of EPA and DHA reported in Table 2 [24]. Greater consumption of omega-3 PUFA is positively correlated with HDL cholesterol, and negatively correlated with TG, resulting in a reduction of developing CVD and CHD [50,57,58]. Results in Table 5, however, indicate omega-3 PUFA, EPA, and DHA, displayed significant positive contributions to TG, LDL, and total cholesterol levels which differs to the negative correlations commonly seen in other studies and as reported by the European Food Safety Authority [53,57]. As we reported larger mean concentrations for nearly the entire FA pool profiled compared to that of a very healthy cohort seen in Abdelmagid et al. [6], high concentrations of health promoting FA and lipid biomarkers may be influenced by increased concentrations of all other FA. We posit the increased omega-3 PUFA concentrations seen are still imparting beneficial health effects despite the positive association to TG and cholesterol. This would provide reasoning for the relatively healthy values of TG and cholesterol seen in Table 1, although mean total FA concentrations were similar to that of an obese cohort [7]. By further establishing strong correlations and determining the unique variance between validated biomarkers and FA concentrations, more precise clinical recommendations can be made to reduce chronic disease risk.

Further work is necessary to establish cut-off values of individual FA concentrations that are deemed to be deficient or in excess. A large body of evidence from multiple cohorts across multiple countries is necessary to establish baseline or "normal" values, and whether region, sex, and/or age specific ranges are necessary [59]. In Table 6, we found that participants with concentrations in the tail ends of FA ranges ( $\geq 95$ th percentile and $\leq 5$ th percentile) also exceeded current lipid biomarker cut-offs for TG. However, based on the sample size of our study, the $\geq 95$ th percentile and $\leq 5$ th percentile contained only 24 participants each, not enough to account for the total participants who exceeded LDL or, total cholesterol cut-offs, thus we expanded the fatty acid cutoff to the $\geq 75$ th percentile which includes 119 participants. Of the 92 participants who exceeded the cut-off for LDL cholesterol, $\sim 70 \%$ of the participants also had FA concentrations in the $\geq 75$ th percentile for PA and SA (Table 6). The exploratory findings in Table 6 and the values seen in Table 5 show that FA concentrations outside the normal range exhibit a large overlap in those with lipid-related health risks. Overall, we more often found a higher proportion of participants who exceeded a lipid biomarker cut-off also having a FA concentration in the $\geq 95$ th percentile compared to those in the $\leq 5$ th percentile. At this point in time, however, we cannot confidently make any conclusions for specific cutoff values designating adequate/inadequate status of individual FA concentrations. FA reference ranges will need to be further profiled in multiple cohorts to assess whether our results are generalizable to the population at large, or specifically Singaporean adults. 


\subsection{Sex Differences}

Along with anthropometric measures, we examined concentrations of circulating FA between sexes, (Tables 1 and 3). Although Singapore consists of a unique ethnic make-up, where cultural differences can influence dietary habits [10], our population was $>90 \%$ Singaporean Chinese and thus, ethnic differences were not evaluated. Based on the World Health Organization Global Status Report (40) the BMI and fasting blood glucose of both males and females in this cohort were below national averages, but males were considered to be overweight $\left(23.3 \mathrm{~kg} / \mathrm{m}^{2}\right)$ based on the BMI cut-offs for Asian populations [46].

We observed mean values for BMI and WC to be below the risk cut-off of an Asian population for developing CVD, diabetes, and dyslipidemia in both sexes, but a number of participants in the higher percentiles shown in Table 4 fell into a risk category [60]. Sexspecific differences in FA status have been shown on a number of occasions, with our results showing women exhibiting significantly greater FA concentrations in Table 3 [35,61-63]. In Abdelmagid et al. [6] when males and females were compared PAO, ALA, and DHA were also all significantly greater except, males had greater concentrations of ALA. In terms of percent composition, Glew et al. [30] found no significant difference between males and females in any of the ten FA highlighted in our study. Independent of dietary intake, females have exhibited a greater genetic capacity to endogenously produce omega-3 PUFA [64]. The use of oral contraceptives in some studies have found to further impact PUFA metabolism, but more recent studies found no significant effect [65,66]. Additionally, pregnant and breastfeeding females have obvious metabolic differences and subsequent nutritional needs compared to males which has also been found to modify FA status [67]. Our study excluded female participants who were taking oral contraceptives and who were pregnant or breastfeeding during data collection. Although omega-3 PUFA were significantly greater in females in this study, the mean FA concentration between sexes was not significantly different (see Table 1). As participants were nearly all the same ethnicity and from the same geographical area, dietary habits were likely similar. Genetic differences between sexes may account for some of the variability shown, but dietary consumption still remains the strongest individual modifier of FA concentrations.

\subsection{Limitations}

This study is limited by a lack of dietary data and measures of physical activity recorded. Both physical activity and dietary consumption, particularly of fat, are modifiers of serum FA concentrations. Inclusion of these two variables in the linear regression model would have provided a more sensitive analysis for assessing the unique variance of FA concentrations to lipid biomarkers. Including all ten FA in the linear regression model violated the collinearity tolerance and VIF, which dietary data may have provided a better understanding as to why specific FA were related due to which foods were consumed.

\section{Conclusions}

In conclusion, our study provides valuable knowledge for the growing desire to develop individual FA reference ranges for assessing chronic disease risk. We are one of the few studies to examine a cohort outside of NA which showcases individual FA concentrations. Inclusion of additional cohorts with very high or low concentrations of FA will help establish a greater understanding of FA levels that may be reflective of chronic disease states.

Author Contributions: Conceptualization, D.W.L.M., C.J.H., and C.A.C.L.; methodology, D.W.L.M. and C.A.C.L.; formal analysis, C.A.C.L.; data curation, X.B.; writing-original draft preparation, C.A.C.L.; writing-review and editing, D.W.L.M., X.B. and C.J.H.; visualization, C.A.C.L. and D.W.L.M.; supervision, D.W.L.M., and C.J.H.; project administration, D.W.L.M.; funding acquisition, D.W.L.M. and C.J.H. All authors have read and agreed to the published version of the manuscript.

Funding: This research was funded by Canadian Institutes of Health Research. 
Institutional Review Board Statement: All procedures involving human participants were approved by the National Healthcare Group Domain Specific Review Board (NHG DSRB, Reference Number: 2013/00783), Singapore and the University of Guelph (Research Ethics Board Number: 18-10-049).

Informed Consent Statement: Informed consent was obtained from all subjects involved in the study.

Data Availability Statement: The data presented in this study is available on request from the corresponding author on reasonable request. The data is not publicly available due to privacy or ethical restrictions.

Conflicts of Interest: The authors declare no conflict of interest.

\section{References}

1. Ratnayake, W.M.N.; Galli, C. Fat and Fatty Acid Terminology, Methods of Analysis and Fat Digestion and Metabolism: A Background Review Paper. Ann. Nutr. Metab. 2009, 55, 8-43. [CrossRef] [PubMed]

2. Fahy, E.; Subramaniam, S.; Brown, H.A.; Glass, C.K.; Merrill, A.H., Jr.; Murphy, R.C.; Raetz, C.R.H.; Russell, D.W.; Seyama, Y.; Shaw, W.; et al. A comprehensive classification system for lipids. J. Lipid Res. 2005, 46, 839-861. [CrossRef] [PubMed]

3. Billingsley, H.E.; Carbone, S.; Lavie, C.J. Dietary Fats and Chronic Noncommunicable Diseases. Nutrients 2018, 10, 1385. [CrossRef] [PubMed]

4. Anderson, T.J.; Grégoire, J.; Pearson, G.J.; Barry, A.R.; Couture, P.; Dawes, M.; Francis, G.A.; Genest, J.; Grover, S.; Gupta, M.; et al. 2016 Canadian Cardiovascular Society Guidelines for the Management of Dyslipidemia for the Prevention of Cardiovascular Disease in the Adult. Can. J. Cardiol. 2016, 32, 1263-1282. [CrossRef] [PubMed]

5. Brenna, J.T.; Plourde, M.; Stark, K.D.; Jones, P.J.; Lin, Y.-H. Best practices for the design, laboratory analysis, and reporting of trials involving fatty acids. Am. J. Clin. Nutr. 2018, 108, 211-227. [CrossRef] [PubMed]

6. Abdelmagid, S.A.; Clarke, S.E.; Nielsen, D.E.; Badawi, A.; El-Sohemy, A.; Mutch, D.M.; Ma, D.W.L. Comprehensive Profiling of Plasma Fatty Acid Concentrations in Young Healthy Canadian Adults. PLoS ONE 2015, 10, e0116195. [CrossRef] [PubMed]

7. Sergeant, S.; Ruczinski, I.; Ivester, P.; Lee, T.C.; Morgan, T.M.; Nicklas, B.J.; Mathias, R.A.; Chilton, F.H. Impact of methods used to express levels of circulating fatty acids on the degree and direction of associations with blood lipids in humans. Br. J. Nutr. 2015, 115, 251-261. [CrossRef]

8. Sera, R.K.; McBride, J.H.; Higgins, S.A.; Rodgerson, D.O. Evaluation of reference ranges for fatty acids in serum. J. Clin. Lab. Anal. 1994, 8, 81-85. [CrossRef]

9. Bradbury, K.E.; Skeaff, C.M.; Crowe, F.; Green, T.J.; Hodson, L. Serum Fatty Acid Reference Ranges: Percentiles from a New Zealand National Nutrition Survey. Nutrients 2011, 3, 152-163. [CrossRef]

10. Tan, K.H.X.; Tan, L.W.L.; Sim, X.; Tai, E.S.; Lee, J.J.-M.; Chia, K.S.; Van Dam, R.M. Cohort Profile: The Singapore Multi-Ethnic Cohort (MEC) study. Int. J. Epidemiol. 2018, 47, 699-699j. [CrossRef]

11. Whitton, C.; Ma, Y.; Bastian, A.C.; Chan, M.F.; Chew, L. Fast-food consumers in Singapore: Demographic profile, diet quality and weight status. Public Heal. Nutr. 2013, 17, 1805-1813. [CrossRef]

12. Henry, C.J.; Kaur, B.; Quek, R.Y.C. Are Asian foods as "fattening" as western-styled fast foods? Eur. J. Clin. Nutr. 2019, 74, 348-350. [CrossRef] [PubMed]

13. Koushki, M.; Nahidi, M.; Cheraghali, F. Physico-chemical properties, fatty acid profile and nutrition in palm oil. J. Paramed. Sci. 2015, 6, 117-134.

14. Jiang, Y.-W.; Sheng, L.-T.; Pan, X.-F.; Feng, L.; Yuan, J.-M.; Pan, A.; Koh, W.-P. Midlife Dietary Intakes of Monounsaturated Acids, n-6 Polyunsaturated Acids, and Plant-Based Fat Are Inversely Associated with Risk of Cognitive Impairment in Older Singapore Chinese Adults. J. Nutr. 2019, 150, 901-909. [CrossRef] [PubMed]

15. Seah, J.Y.H.; Ong, C.N.; Koh, W.-P.; Yuan, J.-M.; Van Dam, R.M. A Dietary Pattern Derived from Reduced Rank Regression and Fatty Acid Biomarkers Is Associated with Lower Risk of Type 2 Diabetes and Coronary Artery Disease in Chinese Adults. J. Nutr. 2019, 149, 2001-2010. [CrossRef]

16. Brostow, D.P.; O Odegaard, A.; Koh, W.-P.; Duval, S.; Gross, M.D.; Yuan, J.; A Pereira, M. Omega-3 fatty acids and incident type 2 diabetes: The Singapore Chinese Health Study. Am. J. Clin. Nutr. 2011, 94, 520-526. [CrossRef]

17. Butler, L.M.; Yuan, J.; Huang, J.Y.; Su, J.; Wang, R.; Koh, W.-P.; Ong, C.-N. Plasma fatty acids and risk of colon and rectal cancers in the Singapore Chinese Health Study. NPJ Precis. Oncol. 2017, 1, 1-10. [CrossRef]

18. Gago-Dominguez, M.; Yuan, J.; Sun, C.-L.; Lee, H.-P.; Yu, M.C. Opposing effects of dietary n-3 and n-6 fatty acids on mammary carcinogenesis: The Singapore Chinese Health Study. Br. J. Cancer 2003, 89, 1686-1692. [CrossRef]

19. Koh, W.-P.; Dan, Y.Y.; Goh, G.B.-B.; Jin, A.; Wang, R.; Yuan, J.-M. Dietary fatty acids and risk of hepatocellular carcinoma in the Singapore Chinese health study. Liver Int. 2015, 36, 893-901. [CrossRef]

20. Sun, Y.; Koh, H.W.L.; Choi, H.; Koh, W.-P.; Yuan, J.-M.; Newman, J.W.; Su, J.; Fang, J.; Ong, C.N.; Van Dam, R.M. Plasma fatty acids, oxylipins, and risk of myocardial infarction: The Singapore Chinese Health Study. J. Lipid Res. 2016, 57, 1300-1307. [CrossRef]

21. Soh, A.Z.; Chee, C.B.; Wang, Y.-T.; Yuan, J.-M.; Koh, W.-P. Dietary Cholesterol Increases the Risk whereas PUFAs Reduce the Risk of Active Tuberculosis in Singapore Chinese. J. Nutr. 2016, 146, 1093-1100. [CrossRef] [PubMed] 
22. Stern, M.C.; Butler, L.M.; Corral, R.; Joshi, A.D.; Yuan, J.-M.; Koh, W.-P.; Yu, M.C. Polyunsaturated Fatty Acids, DNA Repair Single Nucleotide Polymorphisms and Colorectal Cancer in the Singapore Chinese Health Study. J. Nutr. Nutr. 2009,2 , $273-279$. [CrossRef] [PubMed]

23. Sun, Y.; Koh, W.-P.; Yuan, J.; Choi, H.; Su, J.; Ong, C.N.; Van Dam, R.M. Plasma $\alpha$-Linolenic and Long-Chain $\omega$-3 Fatty Acids Are Associated with a Lower Risk of Acute Myocardial Infarction in Singapore Chinese Adults. J. Nutr. 2015, 146, 275-282. [CrossRef]

24. Micha, R.; Khatibzadeh, S.; Shi, P.; Fahimi, S.; Lim, S.; Andrews, K.G.; E Engell, R.; Powles, J.; Ezzati, M.; Mozaffarian, D.; et al. Global, regional, and national consumption levels of dietary fats and oils in 1990 and 2010: A systematic analysis including 266 country-specific nutrition surveys. BMJ 2014, 348, g2272. [CrossRef] [PubMed]

25. Li, S.-W.; Wang, J.; Yang, Y.; Ouyang, S.; Cheng, L.; Liu, H.-Y.; Ma, P.; Luo, W.; Liu, S. Polymorphisms in FADS1 and FADS2 alter plasma fatty acids and desaturase levels in type 2 diabetic patients with coronary artery disease. J. Transl. Med. 2016, 14, 79. [CrossRef] [PubMed]

26. Bi, X.; Loo, Y.T.; Henry, C.J. Body Fat Measurements in Singaporean Adults Using Four Methods. Nutr. 2018, 10, 303. [CrossRef] [PubMed]

27. Bi, X.; Loo, Y.T.; Henry, C.J. Ultrasound measurement of intraabdominal fat thickness as a predictor of insulin resistance and low HDL cholesterol in Asians. Nutrition 2018, 56, 99-103. [CrossRef]

28. Folch, J.; Lees, M.; Sloane Stanley, G. A simple method for the isolation and purification of total lipides from animal tissues. J. Biol. Chem. 1987, 55, 999-1033.

29. Tai, E.S.; Chia, B.L.; Bastian, A.C.; Chua, T.; Ho, S.C.W.; Koh, T.S.; Low, L.P.; Tey, J.S.; Poh, K.-K.; Tan, C.E.; et al. Ministry of Health Clinical Practice Guidelines: Lipids. Singap. Med. J. 2017, 58, 155-166. [CrossRef]

30. VanderJagt, D.J.; Glew, R.H.; Chuang, L.-T.; Berry, T.; Okolie, H.; Crossey, M.J. Lipid Profiles and trans Fatty Acids in Serum Phospholipids of Semi-nomadic Fulani in Northern Nigeria. J. Heal. Popul. Nutr. 2010, 28, 159-166. [CrossRef]

31. A Schwertner, H.; Mosser, E.L. Comparison of lipid fatty acids on a concentration basis vs weight percentage basis in patients with and without coronary artery disease or diabetes. Clin. Chem. 1993, 39, 659-663. [CrossRef] [PubMed]

32. Whitton, C.; Neelakantan, N.; Ong, C.N.; Van Dam, R.M. Reproducibility of Dietary Biomarkers in a Multiethnic Asian Population. Mol. Nutr. Food Res. 2019, 63, e1801104. [CrossRef]

33. Asselin, C.; Ducharme, A.; Ntimbane, T.; Ruiz, M.; Fortier, A.; Guertin, M.-C.; Lavoie, J.; Diaz, A.; Levy, É.; Tardif, J.-C.; et al. Circulating levels of linoleic acid and HDL-cholesterol are major determinants of 4-hydroxynonenal protein adducts in patients with heart failure. Redox Biol. 2014, 2, 148-155. [CrossRef]

34. Kang, M.; Lee, A.; Yoo, H.J.; Kim, M.; Kim, M.; Shin, D.Y.; Lee, J.H. Association between increased visceral fat area and alterations in plasma fatty acid profile in overweight subjects: A cross-sectional study. Lipids Heal. Dis. 2017, 16, 248. [CrossRef] [PubMed]

35. Tremblay-Franco, M.; Zerbinati, C.; Pacelli, A.; Palmaccio, G.; Lubrano, C.; Ducheix, S.; Guillou, H.; Iuliano, L. Effect of obesity and metabolic syndrome on plasma oxysterols and fatty acids in human. Steroids 2015, 99, 287-292. [CrossRef] [PubMed]

36. Pickens, C.A.; Sordillo, L.M.; Comstock, S.S.; Harris, W.S.; Hortos, K.; Kovan, B.; Fenton, J.I. Plasma phospholipids, non-esterified plasma polyunsaturated fatty acids and oxylipids are associated with BMI. Prostaglandins Leukot. Essent. Fat. Acids 2015, 95, 31-40. [CrossRef]

37. Léveillé, P.; Chouinard-Watkins, R.; Windust, A.; Lawrence, P.; Cunnane, S.C.; Brenna, J.T.; Plourde, M. Metabolism of uniformly labeled 13 C-eicosapentaenoic acid and 13 C-arachidonic acid in young and old men. Am. J. Clin. Nutr. 2017, 106, 467-474. [CrossRef]

38. Sun, Y.; Neelakantan, N.; Wu, Y.; Lote-Oke, R.; Pan, A.; Van Dam, R.M. Palm Oil Consumption Increases LDL Cholesterol Compared with Vegetable Oils Low in Saturated Fat in a Meta-Analysis of Clinical Trials. J. Nutr. 2015, 145, 1549-1558. [CrossRef]

39. Stark, K.D.; Van Elswyk, M.E.; Higgins, M.R.; Weatherford, C.A.; Salem, J.N. Global survey of the omega-3 fatty acids, docosahexaenoic acid and eicosapentaenoic acid in the blood stream of healthy adults. Prog. Lipid Res. 2016, 63, 132-152. [CrossRef]

40. World Health Organization. Global Status Report on Noncommunicable Diseases 2014; World Health Organization: Geneva, Switzerland, 2014.

41. Lee, Y.S.; Biddle, S.; Chan, M.F.; Cheng, A.; Cheong, M.; Chong, Y.S.; Foo, L.L.; Lee, C.H.; Lim, S.C.; Ong, W.S.; et al. Health Promotion Board-Ministry of Health Clinical Practice Guidelines: Obesity. Singap. Med. J. 2015, 57, 292-300. [CrossRef]

42. Kim, C.-H.; Park, H.S.; Park, M.; Kim, H.; Kim, C. Optimal cutoffs of percentage body fat for predicting obesity-related cardiovascular disease risk factors in Korean adults. Am. J. Clin. Nutr. 2011, 94, 34-39. [CrossRef]

43. Deurenberg-Yap, M.; Chew, S.K.; Deurenberg, P. Elevated body fat percentage and cardiovascular risks at low body mass index levels among Singaporean Chinese, Malays and Indians. Obes. Rev. 2002, 3, 209-215. [CrossRef] [PubMed]

44. Deurenberg-Yap, M.; Schmidt, G.; A Van Staveren, W.; Deurenberg, P. The paradox of low body mass index and high body fat percentage among Chinese, Malays and Indians in Singapore. Int. J. Obes. 2000, 24, 1011-1017. [CrossRef] [PubMed]

45. Barba, C.; Cavalli-Sforza, T.; Cutter, J.; Deurenberg, P.; Deurenberg-Yap, M.; Darnton-Hill, I.; Gill, T.; James, P.; Ko, G.; Miu, A.H.; et al. Appropriate body-mass index for Asian populations and its implications for policy and intervention strategies. Lancet 2004, 363, 157-163. [CrossRef]

46. Jih, J.; Mukherjea, A.; Vittinghoff, E.; Nguyen, T.T.; Tsoh, J.Y.; Fukuoka, Y.; Bender, M.S.; Tseng, W.; Kanaya, A.M. Using appropriate body mass index cut points for overweight and obesity among Asian Americans. Prev. Med. 2014, 65, 1-6. [CrossRef]

47. Clandinin, M.T.; Cook, S.L.; Konard, S.D.; French, M.A. The effect of palmitic acid on lipoprotein cholesterol levels. Int. J. Food Sci. Nutr. 2000, 51, s61-s71. [CrossRef] 
48. Wendland, E.; Farmer, A.; Glasziou, P.; Neil, A. Effect of $\alpha$ linolenic acid on cardiovascular risk markers: A systematic review. Heart 2006, 92, 166-169. [CrossRef]

49. Hiraoka-Yamamoto, J.; Ikeda, K.; Negishi, H.; Mori, M.; Hirose, A.; Sawada, S.; Onobayashi, Y.; Kitamori, K.; Kitano, S.; Tashiro, M.; et al. Serum lipid effects of a monounsaturated (palmitoleic) fatty acid-rich diet based on macadamia nuts in healthy, young Japanese women. Clin. Exp. Pharmacol. Physiol. 2004, 31, S37-S38. [CrossRef]

50. Leslie, M.A.; Cohen, D.J.A.; Liddle, D.M.; Robinson, L.E.; Ma, D.W.L. A review of the effect of omega-3 polyunsaturated fatty acids on blood triacylglycerol levels in normolipidemic and borderline hyperlipidemic individuals. Lipids Heal. Dis. 2015, 14, 1-18. [CrossRef]

51. Williams, C.M.; Salter, A. Saturated fatty acids and coronary heart disease risk: The debate goes on. Curr. Opin. Clin. Nutr. Metab. Care 2016, 19, 97-102. [CrossRef]

52. Triglycerides: Why do they matter? Mayo Clinic. Available online: https://www.mayoclinic.org/diseases-conditions/highblood-cholesterol/in-depth/triglycerides/art-20048186. (accessed on 7 June 2020).

53. Grimsgaard, S.; Bønaa, K.H.; Bjerve, K.S. Fatty acid chain length and degree of unsaturation are inversely associated with serum triglycerides. Lipids 2000, 35, 1185-1193. [CrossRef] [PubMed]

54. DiNicolantonio, J.J.; O'Keefe, J.H. Effects of dietary fats on blood lipids: A review of direct comparison trials. Open Hear. 2018, 5, e000871. [CrossRef] [PubMed]

55. Talayero, B.G.; Sacks, F.M. The Role of Triglycerides in Atherosclerosis. Curr. Cardiol. Rep. 2011, 13, 544-552. [CrossRef] [PubMed]

56. Alexopoulos, A.-S.; Qamar, A.; Hutchins, K.; Crowley, M.J.; Batch, B.C.; Guyton, J.R. Triglycerides: Emerging Targets in Diabetes Care? Review of Moderate Hypertriglyceridemia in Diabetes. Curr. Diabetes Rep. 2019, 19, 13. [CrossRef] [PubMed]

57. Panel, E.; Nda, A. Scientific Opinion on the substantiation of health claims related to eicosapentaenoic acid (EPA), docosahexaenoic acid (DHA), docosapentaenoic acid (DPA) and maintenance of normal cardiac function (ID 504, 506, 516, 527, 538, 703, 1128, 1317, 1324, 1325). EFSA J. 2010, 8, 10.

58. Arca, M.; Borghi, C.; Pontremoli, R.; De Ferrari, G.; Colivicchi, F.; Desideri, G.; Temporelli, P. Hypertriglyceridemia and omega-3 fatty acids: Their often overlooked role in cardiovascular disease prevention. Nutr. Metab. Cardiovasc. Dis. 2018, 28, 197-205. [CrossRef] [PubMed]

59. Häggström, M. Establishment and clinical use of reference ranges. WikiJournal Med. 2014, 1, 1-7. [CrossRef]

60. Lam, B.C.C.; Koh, G.C.H.; Chen, C.; Wong, M.T.K.; Fallows, S.J. Comparison of Body Mass Index (BMI), Body Adiposity Index (BAI), Waist Circumference (WC), Waist-To-Hip Ratio (WHR) and Waist-To-Height Ratio (WHtR) as predictors of cardiovascular disease risk factors in an adult population in Singapore. PLOS ONE 2015, 10, e0122985.

61. Hodson, L.; Eyles, H.C.; McLachlan, K.J.; Bell, M.L.; Green, T.J.; Skeaff, C.M. Plasma and Erythrocyte Fatty Acids Reflect Intakes of Saturated and n-6 PUFA within a Similar Time Frame. J. Nutr. 2013, 144, 33-41. [CrossRef]

62. Tsou, P.; Wu, C.-J. Sex-Dimorphic Association of Plasma Fatty Acids with Cardiovascular Fitness in Young and Middle-Aged General Adults: Subsamples from NHANES 2003. Nutrients 2018, 10, 1558. [CrossRef]

63. Lohner, S.; Fekete, K.; Marosvölgyi, T.; Decsi, T. Gender Differences in the Long-Chain Polyunsaturated Fatty Acid Status: Systematic Review of 51 Publications. Ann. Nutr. Metab. 2013, 62, 98-112. [CrossRef] [PubMed]

64. Burdge, G.C.; Wootton, S.A. Conversion of $\alpha$-linolenic acid to eicosapentaenoic, docosapentaenoic and docosahexaenoic acids in young women. Br. J. Nutr. 2002, 88, 411-420. [CrossRef] [PubMed]

65. Fehily, A.M.; Dickerson, J.W.; Meade, B.W.; Ellis, F.R. Plasma and erythrocyte membrane fatty acids in oral contraceptive users. Clin. Chim. Acta 1982, 120, 41-47. [CrossRef]

66. Jensen, M.D.; Levine, J. Effects of oral contraceptives on free fatty acid metabolism in women. Metabolism 1998, 47, 280-284. [CrossRef] [PubMed]

67. Marangoni, F.; Cetin, I.; Verduci, E.; Canzone, G.; Giovannini, M.; Scollo, P.; Corsello, G.; Poli, A. Maternal Diet and Nutrient Requirements in Pregnancy and Breastfeeding. An Italian Consensus Document. Nutrients 2016, 8, 629. [CrossRef] [PubMed] 